\title{
Expression of IMP3 as a marker for predicting poor outcome in gastroenteropancreatic neuroendocrine neoplasms
}

\author{
LI-MIAN ER ${ }^{1}$, YONG LI $^{2}$, MING-LI WU ${ }^{1}$, QUN ZHAO ${ }^{2}$, BI-BO TAN $^{2}$, XIAO-LING WANG ${ }^{3}$ and SHI-JIE WANG $^{1}$ \\ ${ }^{1}$ Department of Endoscopy; ${ }^{2}$ The Third Department of Surgery; ${ }^{3}$ Department of Pathology, \\ The Fourth Hospital of Hebei Medical University, Shijiazhuang, Hebei 050011, P.R. China
}

Received August 27, 2015; Accepted November 30, 2016

DOI: $10.3892 / \mathrm{ol} .2017 .5735$

\begin{abstract}
The aim of the present study was to investigate the expression and clinical significance of oncofetal protein insulin-like growth factor (IGF) II mRNA-binding protein 3 (IMP3) in the differentiation of gastroenteropancreatic neuroendocrine neoplasm (GEP-NEN). A total of 162 patients who were diagnosed with GEP-NEN, and who underwent surgical or endoscopic resection from January 2006 to March 2013, were enrolled in the study, including 85 cases of grade (G)1 neuroendocrine tumors, 40 cases of G2 neuroendocrine tumors, 28 cases of G3 neuroendocrine carcinomas and 9 cases of mixed stage adenoneuroendocrine carcinomas. The clinical and pathological data were recorded for analysis. The expression of IMP3, cluster of differentiation (CD)44, IGF1 receptor (IGF1R) and matrix metalloproteinase (MMP)2 was determined by immunohistochemistry. SPSS 13.0 software was used for data processing and analyses, and $\mathrm{P}<0.05$ was used to determine significance. Oncofetal protein IMP3 exhibited a high expression rate (74.69\%) in GEP-NEN. IMP3-positive cases demonstrated significantly decreased overall and disease-free survival times, as compared with IMP3-negative cases $(\mathrm{P}=0.012)$. Overexpression of IMP3 was correlated with tumor grade, clinical stage, tumor size and poor prognosis (all $\mathrm{P}<0.05)$. Therefore, patients with overexpressed IMP3 had a poorer prognosis $(\mathrm{P}<0.01)$; COX regression analysis revealed that the overexpression of IMP3, the tumor grade, tumor size and metastasis of GEP-NEN were each associated with the clinical outcomes. The results also indicated that the expression rates of CD44, IGF1R and MMP2 in GEP-NEN were $19.75,53.7$ and $55.56 \%$, respectively. While it was negatively associated with the expression of CD44 ( $\mathrm{r}=-0.131$; $\mathrm{P}=0.096$ ), the expression of IMP3 was positively correlated
\end{abstract}

Correspondence to: Professor Yong Li, The Third Department of Surgery, The Fourth Hospital of Hebei Medical University, 12 Jiankang Road, Shijiazhuang, Hebei 050011, P.R. China

E-mail: china.li_yong_hbth@126.com

Key words: insulin-like growth factor II mRNA binding protein 3, gastroenteropancreatic neuroendocrine neoplasms, immunohistochemistry, predictor, prognosis with the expression of IGF1R and MMP2 $(\mathrm{r}=0.288, \mathrm{P}<0.01$; $\mathrm{r}=0.208, \mathrm{P}=0.008)$. In addition, the expression levels of IGF1R and MMP2 were positively associated $(r=0.687 ; \mathrm{P}<0.01)$. In conclusion, high IMP3 expression levels were determined to be associated with a high disease stage in patients with GEP-NEN, thus it may serve as a predictor for metastasis and poor clinical outcomes in GEP-NEN.

\section{Introduction}

The incidence of gastroenteropancreatic neuroendocrine neoplasms (GEP-NENs) has increased over the last few decades $(1,2)$. The clinical course of the disease is almost indolent (3). Although $\leq 2 / 3$ of patients are diagnosed with GEP-NEN and distant metastases, the five-year survival rate exceeds $60 \%$ (4). For this reason, the incidence of GEP-NEN is higher than that of pancreatic, gastric and esophageal adenocarcinomas, making it the second most prevalent type of cancer of the gastrointestinal (GI) tract (5). Early detection and subsequent effective therapy may improve the prognosis of GEP-NEN.

Insulin-like growth factor (IGF) II mRNA-binding protein 3 (IMP3) is a member of the IMP family, which consists of IMP1, IMP2 and IMP3 (6). IMPs bind to and influence the transportation, localization and stability of target mRNA, particularly during the early stages of human and mouse embryogenesis (7). IMP3 is located on chromosome 7p11.5 and encodes a 4,350 bp mRNA (8). IMP3 is expressed in the developing epithelium, muscle and placenta during the early stages of human and mouse embryogenesis, and low or undetectable levels of IMP3 are present in adult tissues (9). IMP3 has been demonstrated to be overexpressed in gastric cancer, colon cancer and adenocarcinoma of the lung (10-12). The expression of IMP3 was identified to correlate with aggressive tumor progression, suggesting that IMP3 may have a role in tumor invasion and metastasis.

Previous studies reported that the oncogenic effects of are mediated through IGF-II, an mRNA that is activated by IMP3 $(13,14)$. IGF2 ligands activate a common receptor, the IGF1 receptor (IGF1R), which signals mitogenic, anti-apoptotic and transforming activities (15). The IGF1R is a cell-surface tyrosine kinase receptor coupled to several intracellular secondary messenger signaling pathways, including the rat sarcoma/rapidly accelerated fibrosarcoma/mitogen 
activated protein kinase and the phosphatidylinositol-3 kinase/protein kinase B signaling cascades (16). The IGF1R is vital for cell survival, as illustrated by the development of a malignant phenotype in which the IGFIR gene is disrupted in mice (17). A recent study reported that deletion of the mouse IMP3 gene, leads to cluster of differentiation (CD) 44 mRNA downregulation (18). CD44 is a cell adhesion molecule that is able to promote the degradation of type IV collagen fibers and combine with the cytoskeletal protein Ezrin/radixin/moesin; the downregulation of CD44 may reduce the adhesion between the cell and the matrix (18).

To date, and to the best of our knowledge, no study into the independent prognostic value of IMP3 in GEP-NEN has been reported. To determine the involvement of IMP3 in the prognosis of GEP-NEN, 162 cases of GEP-NEN were retrospectively evaluated by detecting the expression of IMP 3 in a series of GEP-NENs, in addition to the correlation between the invasion-associated proteins matrix metalloproteinase (MMP) 2, IGF1R, CD44 and clinical outcomes.

\section{Materials and methods}

Patients. The current study enrolled 162 patients with diagnosed GEP-NEN, who had undergone endoscopic or surgical resection at The Fourth Hospital of Hebei Medical University (Shijiazhuang, China) from September 2006 to March 2013. The patients had no evidence of any other malignancies, and those who had received preoperative chemotherapy or radiotherapy were excluded. The clinical data on gender, age, depth of tumor invasion, location and tumor size were obtained from the medical records, and the data on histological differentiation and the extent of lymph node involvement were obtained from the pathological reports. The Ethical Committee of The Fourth Hospital of Hebei Medical University approved the study protocol, and all patients, prior to surgical gastrectomy or endoscopic resection, provided written informed consent regarding surgical specimens. Immunohistochemical staining was performed in order to analyze the expression of IMP3 in primary GEP-NEN tissues. Follow-up data were recorded from all the patients until December 2014. The mean follow-up time was 35.15 months and the methods of follow-up included telephone calls, e-mail and outpatient visiting.

GEP-NEN diagnosis and classification. According to the recent World Health Organization (WHO) classification (18), the tumors were classified as grade $(\mathrm{G}) 1$ neuroendocrine tumors (G1 NETs), G2 NETs and G3 neuroendocrine carcinoma, large-cell (G3 NEC-LC) or small-cell (G3 NEC-SC) subtypes. Certain features, including the mitotic index, Ki-67 labeling index, tumor size and the presence of necrosis or blood vessel invasion were required for tumor classification (Table I) (19).

Immunohistochemical staining. The surgically resected tissue samples were formaldehyde-fixed (10\%) and paraffin-embedded. The paraffin blocks were cut serially into $4-\mu \mathrm{m}$ thick sections. The sections were deparaffinized with xylene and rehydrated in a series of alcohols. The samples were incubated at room temperature in $3 \% \mathrm{H}_{2} \mathrm{O}_{2}$ for $15 \mathrm{~min}$ to quench any endogenous peroxidase activity, prior to being washed with PBS. Citrate buffer (0.01 M, pH 6.0) was used for antigen retrieval, and normal goat serum (5\%) was used to block the samples at room temperature for $30 \mathrm{~min}$ to. For antigen retrieval, the slides were autoclaved at $110^{\circ} \mathrm{C}$ in citrate buffer (cat. no. MVS-0066; Fuzhou Maxim Biotech, Ltd., Fujian, China) for $2 \mathrm{~min}$, then cooled at room temperature for $10 \mathrm{~min}$ and washed with PBS twice. The sections were incubated overnight at $4^{\circ} \mathrm{C}$ with the following primary antibodies: Mouse anti-human IMP3 monoclonal antibody (cat. no. YM0374; 1:200), rabbit anti-human IGF1R (cat. no. YT2282; 1:400), anti-MMP2 polyclonal antibody (cat. no. YT2798; 1:100) (all ImmunoWay Biotechnology Company, DE, USA) and rabbit anti-human CD44 polyclonal antibody (cat. no. ZA-0537; 1:60; Zhongshan Golden Bridge Biotechnology Co., Ltd., Beijing, China). Following washing in PBS, the sections were incubated with a secondary horseradish-peroxidase labelled polymer antibody (cat. no. PV8000-D; 1:100; Zhongshan Golden Bridge Biotechnology Co., Ltd) at room temperature for $30 \mathrm{~min}$ and then washed with PBS once more. Subsequently, the sections were washed with PBS, stained with diaminobenzidine (DAB) and counterstained with hematoxylin. For the negative control, the primary antibody was substituted with PBS.

Two pathologists blinded to the patient outcomes independently evaluated immunoreactivity. The evaluation was based on the staining intensity and the extent of staining. Positive staining for IMP3 was defined as a dark brown cytoplasmic staining pattern in the tumor cells observed at low-power magnification (x10); Tumors were regarded as immune-positive if $>30 \%$ of tumor cells showed immunoreactivity. Samples were considered positive for CD44 and IGF1R if brown granules appeared in the plasma membrane, and positive for MMP2 if brown granules appeared in the cytoplasm. Tumors were regarded as immune-positive if $>10 \%$ of tumor cells showed immunoreactivity.

Statistical analysis. The $\chi^{2}$, Wilcoxon signed rank tests and Spearman's correlation were used to analyze the data. For the analysis of prognosis, the Kaplan-Meier method was employed to calculate the survival rate, and the Cox proportional hazards regression model was used for multivariate analysis. All data were processed using SPSS version 13.0 statistical software (SPSS Inc., Chicago, IL, USA). $\mathrm{P}<0.05$ was considered to indicate a statistically significant difference.

\section{Results}

Clinicopathological features. In total, 162 patients with histologically confirmed sporadic GEP-NEN were retrospectively analyzed. The clinicopathological features are summarized in Table I. A total of $91(56.2 \%)$ patients were male and 71 $(43.8 \%)$ were female. The mean age at initial diagnosis was $54.0 \pm 12.2$ years (range, 19-79 years). Of these, 125 cases were NET (G1 and G2), 28 cases were NEC (G3) and 9 cases were mixed adenoneuroendocrine carcinomas (MANEC). At least one neuroendocrine marker (synaptophysin, chromogranin A or CD56) was positive in each tumor. According to the European Neuroendocrine Tumor Society and American Joint Committee on Cancer tumor-node-metastasis (TNM) staging criteria (20). 60 patients (37.0\%) were stage I, $21(13.0 \%)$ were stage II, 7 (4.3\%) were stage III and $30(18.5 \%)$ were stage IV, 


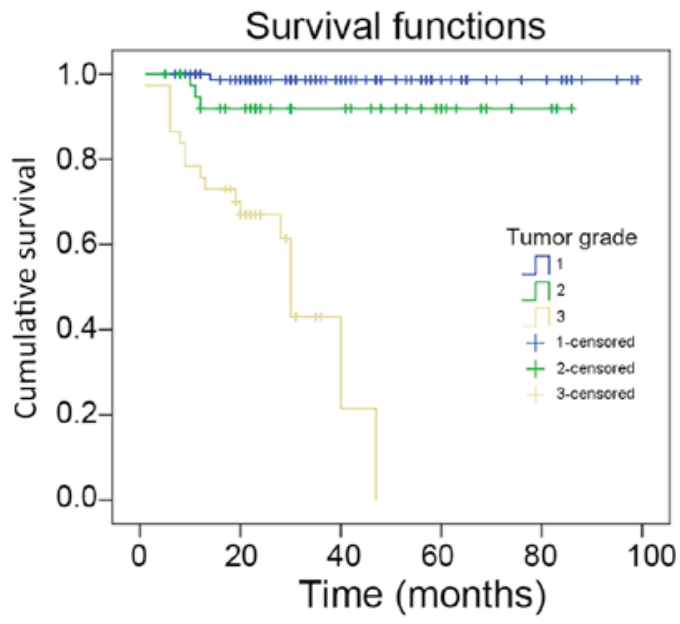

Figure 1. Kaplan-Meier survival curve of patients with gastroenteropancreatic neuroendocrine neoplasm with different tumor grade.

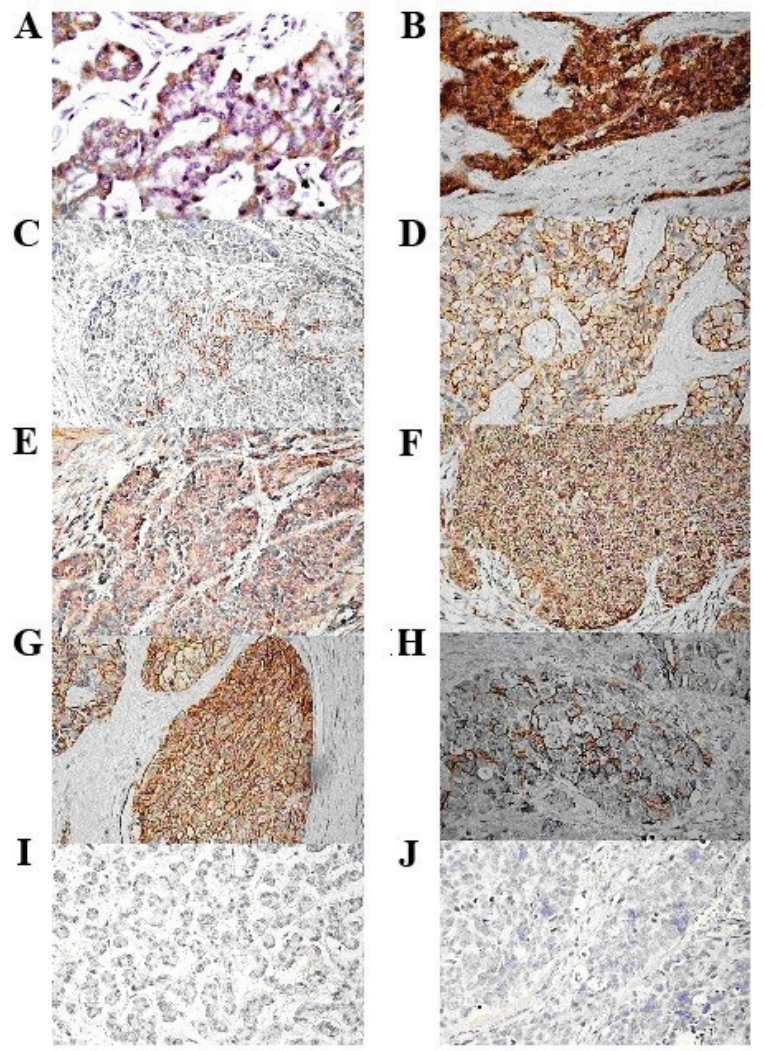

Figure 2. Immunohistochemical staining for IMP3, IGF1R, MMP2 and CD44 in GEP-NET and GEP-NEC. Positive IMP3 staining in (A) GEP-NET and (B) GEP-NEC. Positive IGF1R staining in (C) GEP-NET and (D) GEP-NEC. Positive MMP-2 staining in (E) GEP-NET and (F) GEP-NEC. Positive CD44 staining in (G) GEP-NET and (H) GEP-NEC. Negative IMP3, IGF1R, MMP2 and CD44 staining in (I) GEP-NET and (J) GEP-NEC. Magnification, $x 400$. IMP3, insulin-like growth factor II mRNA-binding protein 3; IGF1R, insulin-like growth factor 1 receptor; MMP2, matrix metalloproteinase 2; CD44, cluster of differentiation 33; GEP-NET, gastroenteropancreatic neuroendocrine tumor; GEP-NEC, gastroenteropancreatic neuroendocrine carcinoma.

A total of $19(42.1 \%)$ patients had distant metastases at the time of diagnosis, Of these, $17(17 / 19,89.5 \%)$ were liver metastases. Of the 162 patients, 148 received long-term follow-up from the

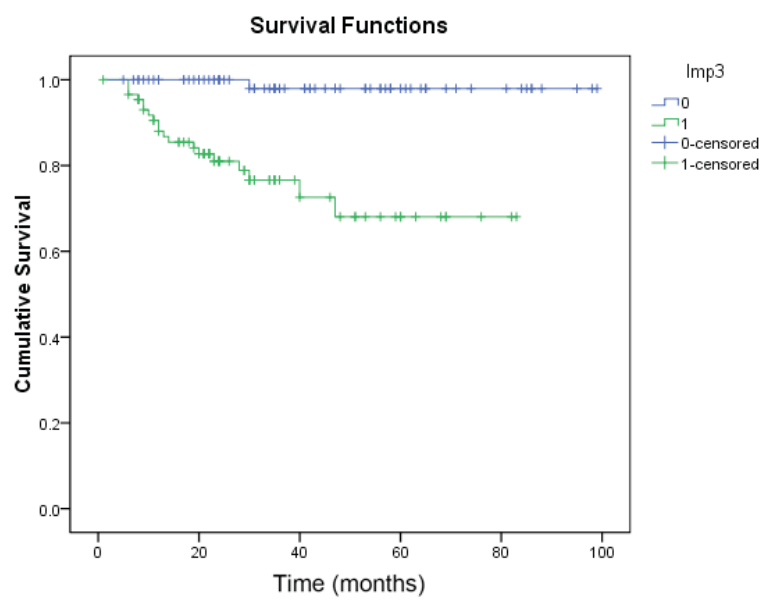

Figure 3. Kaplan-Meier survival curves of patients with gastroenteropancreatic neuroendocrine neoplasm in association with IMP3 expression.

time of diagnosis to February 2015; the median follow-up time was 30 months (range, 1-99 months). Kaplan-Meier analysis was used to analyze the association between the tumor grade and prognosis, and the survival curve is presented in Fig. 1.

Expression of IMP3, IGF1R, MMP2 and CD44 in GEP-NEN. IMP3 was primarily expressed in the cell cytoplasm. The overall expression rate of IMP3 was $74.69 \%$ in the tumor tissue of 162 patients with GEP-NEN. A total of 41 cases $(26.31 \%)$ exhibited negative IMP3 expression. The positive expression rate of IMP3 in NEC and MANEC tissues was 91.9\% (34/37), which was significantly higher than in NET tissues $(69.6 \%$; 87/125; $\mathrm{P}<0.001)$. The positive expression rate of IGF1R, MMP2 and CD44 in GEP-NEN tissue was 56.79\% (92/162), $53.70 \%$ (87/162) and 20.99\% (34/162), respectively (Fig. 2).

Association between IMP3 expression and the clinicopathological characteristics of GEP-NEN. There was no correlation between the expression of IMP3 and the gender $(\mathrm{P}=0.150)$ and age $(\mathrm{P}=0.569)$ of the patients. The expression of IMP3 was correlated with the tumor grade, tumor stage and tumor size; IMP3 expression levels increased with increases in tumor grade and stage $(\mathrm{P}=0.039)$. The positive expression rate of IMP3 in NET (G1 and G2) tissues was 69.6\% (87/125), which was significantly lower compared with that in poorly-differentiated GEP-NEN samples $(91.9 \% ; 34 / 37 ; \mathrm{P}=0.005)$. The IMP3 expression levels in patients with tumor invasion limited to stages T1/T2 $(70.6 \%$; 84/121) were significantly lower, as compared with in patients with tumor invasion of stages T3/T4 $(86.0 \% ; 37 / 43 ; \mathrm{P}=0.046)$. The positive expression rate of IMP3 in patients with GEP-NEN and a tumor size $\leq 2 \mathrm{~cm}$ was lower than in patients with a tumor size $>2 \mathrm{~cm}(\mathrm{P}=0.024)$. The results of immunohistochemical studies into IMP3 expression in GEP-NEN tissues are summarized in Table II.

Significance of IMP3 expression in the prognosis of GEP-NEN. Kaplan-Meier analysis was used to analyze the association between IMP3 expression and prognosis; the survival curve is presented in Fig. 3. The survival rate in patients with positive expression of IMP3 was low, as compared with in patients with 
Table I. The World Health Organization 2010 classification system of GEP-NEN.

\begin{tabular}{lll}
\hline Grade & \multicolumn{1}{c}{ Lung, thymus } & \multicolumn{1}{c}{ GEP-NEN } \\
\hline Low $(\mathrm{G} 1)$ & Carcinoid tumor & Neuroendocrine tumor grade 1 \\
Intermediate (G2) & Atypical carcinoid tumor & Neuroendocrine tumor grade 2 \\
High (G3) & Small cell carcinoma & Neuroendocrine carcinoma grade 3, small-cell carcinoma \\
& & Neuroendocrine carcinoma grade 3, large-cell neuroendocrine carcinoma \\
& Large cell neuroendocrine carcinoma
\end{tabular}

GEP-NEN, gastroenteropancreatic neuroendocrine neoplasm; G, grade.

Table II. Correlation between clinicopathological factors and the expression of IMP3 in GEP-NEN $(n=162)$.

\begin{tabular}{|c|c|c|c|c|c|}
\hline \multirow[b]{2}{*}{ Characteristics } & \multicolumn{2}{|c|}{ IMP3 } & \multirow[b]{2}{*}{ Positive rate $(\%)$} & \multirow[b]{2}{*}{$\chi^{2}$} & \multirow[b]{2}{*}{ P-value } \\
\hline & Positive & Negative & & & \\
\hline \multicolumn{6}{|l|}{ Gender } \\
\hline Male & 72 & 19 & 79.1 & \multirow[t]{2}{*}{2.155} & \multirow[t]{2}{*}{0.150} \\
\hline Female & 49 & 22 & 69.0 & & \\
\hline \multicolumn{6}{|l|}{ Age (years) } \\
\hline$<60$ & 81 & 25 & 66.9 & \multirow[t]{2}{*}{0.482} & \multirow[t]{2}{*}{0.569} \\
\hline$\geq 60$ & 40 & 16 & 61.0 & & \\
\hline \multicolumn{6}{|l|}{ Tumor grade } \\
\hline $\mathrm{G} 1+\mathrm{G} 2$ & 87 & 38 & 69.6 & \multirow[t]{2}{*}{7.505} & \multirow[t]{2}{*}{$0.005^{\mathrm{a}}$} \\
\hline $\mathrm{G} 3+\mathrm{M}$ & 34 & 3 & 91.9 & & \\
\hline \multicolumn{6}{|l|}{ Tumor stage } \\
\hline $\mathrm{T} 1+\mathrm{T} 2$ & 84 & 35 & 70.6 & \multirow[t]{2}{*}{3.993} & \multirow[t]{2}{*}{$0.046^{\mathrm{a}}$} \\
\hline $\mathrm{T} 3+\mathrm{T} 4$ & 37 & 6 & 86.0 & & \\
\hline \multicolumn{6}{|c|}{ Lymphatic metastasis } \\
\hline Negative & 90 & 35 & 72.0 & \multirow[b]{2}{*}{2.097} & \multirow[b]{2}{*}{0.148} \\
\hline Positive & 31 & 6 & 83.8 & & \\
\hline \multicolumn{6}{|l|}{ Metastasis } \\
\hline Negative & 93 & 36 & 72.1 & \multirow[b]{2}{*}{2.262} & \multirow[b]{2}{*}{0.179} \\
\hline Positive & 28 & 5 & 84.8 & & \\
\hline \multicolumn{6}{|l|}{ Tumor size } \\
\hline$<2 \mathrm{~cm}$ & 88 & 35 & 71.5 & \multirow{2}{*}{5.072} & \multirow[t]{2}{*}{$0.024^{\mathrm{a}}$} \\
\hline$\geq 2 \mathrm{~cm}$ & 43 & 6 & 87.8 & & \\
\hline
\end{tabular}

IMP3, insulin-like growth factor (IGF) II mRNA-binding protein 3; GEP-NEN, gastroenteropancreatic neuroendocrine neoplasm; G, grade; $\mathrm{M}$, metastasis.

negative IMP3 expression $(\mathrm{P}<0.001)$. In order to determine the independent risk factors affecting the prognosis of GEP-NEN, Cox risk regression analysis was performed using six indicators as follows: IMP3 expression, tumor grade, lymphatic metastasis, metastasis, tumor size and tumor stage. The results demonstrated that positive IMP3 expression, tumor grade and tumor stage were all independent risk factors affecting the prognosis of patients with GEP-NEN (all Ps<0.05; Table III).

Association between the expression of IMP3 and IGFIR, $M M P 2$, and CD44 in GEP-NEN. Spearman's correlation analysis demonstrated that IMP3 protein expression was positively associated with IGFR1 and MMP2 expression ( $\mathrm{r}=-0.288$,
Table III. Results of the Cox risk analysis model of gastroenteropancreatic neuroendocrine neoplasms.

\begin{tabular}{llcc}
\hline Clinicopathological factor & P-value & OR & $95.0 \%$ CI \\
\hline Tumor grade & $0.006^{\mathrm{a}}$ & 0.139 & $0.034 \sim 0.572$ \\
Lymphatic metastasis & 0.101 & 0.332 & $0.089 \sim 1.239$ \\
Metastasis & $0.002^{\mathrm{a}}$ & 0.193 & $0.068 \sim 0.551$ \\
IMP3 expression & $0.019^{\mathrm{a}}$ & 0.076 & $0.009 \sim 0.649$ \\
Tumor size & $0.040^{\mathrm{a}}$ & 2.757 & $1.048 \sim 7.250$ \\
Tumor stage & 0.876 & 0.790 & $0.041 \sim 15.204$ \\
\hline
\end{tabular}

${ }^{\mathrm{a}} \mathrm{P}<0.05$. OR, odds ratio; $\mathrm{CI}$, confidence interval. 
$\mathrm{P}<0.001 ; \mathrm{r}=0.208, \mathrm{P}=0.008)$, but not with $\mathrm{CD} 44$ expression $(r=-0.131 ; P=0.096)$. The expression of IGF1R and MMP2 were positively correlated $(\mathrm{r}=0.687 ; \mathrm{P}<0.001)$. In addition, the expression of IGFR1 and MMP2 demonstrated no significant association with CD44.

\section{Discussion}

The IMP3 gene, also known as the $\mathrm{K}$ homology domain-containing gene or L523S, encodes the IMP3 protein (7). It is regarded as a novel oncofetal protein that may be re-expressed in various malignant tissues, including pancreatic, lung, kidney and ovarian cancer among others (21-24). The expression of IMP3 was identified to correlate with aggressive tumor progression, suggesting that IMP3 may have a role in tumor invasion and metastasis (25). To the best of our knowledge, the only previous study regarding IMP3 protein expression in adult lung NEC suggests that it is a marker of poor outcome in lung NET; its correlation with NANOG expression suggests the implication of IMP3 in stem cell processes and its association with a Ki67 labeling index of $\geq 4 \%$ stratifies a subset of atypical carcinoids with a higher risk of recurrence and mortality (26). However, to the best of our knowledge, the clinicopathological and prognostic significance of IMP3 expression in GEP-NEN has not previously been reported. Our study systematically examined the expression of IMP3 in 162 cases of GEP-NEN tissues and analyzed its association with the clinical features and prognosis.

The results of the present study demonstrated that IMP3 was highly expressed in GEP-NEN tissues. The survival rate of patients with positive expression of IMP3 was low, as compared with in patients with negative expression. In addition, the overexpression of IMP3 was associated with a high tumor grade, late clinical stage and deep invasion. Furthermore, tumor size was identified to contribute to high IMP3 expression levels, indicating that IMP3 is associated with the development of GEP-NEN and the degree of malignancy; the IMP3 gene was further enhanced during tumor progression and may contribute to the progress of GEP-NEN. The prognostic analysis demonstrated that the three-year survival rate of patients with positive IMP3 expression was significantly lower than for patients with negative expression; therefore, IMP3 is an independent risk factor affecting the prognosis of patients with GEP-NEN. These results demonstrate that IMP3 may have an important role as an oncogene in the development of GEP-NEN, and may be considered as a potential tumor marker and a predictor of prognosis in the diagnosis and treatment of GEP-NEN.

Pasiliao et al (27) identified that the observed impairment of pancreatic cancer cell behavior following IMP3 depletion is likely due to inhibition of the CD44-RhoA signaling cascade. IMP3 belongs to the family of IGFII-mRNA binding proteins that affect RNA stability and influence cell growth and migration during embryonic development (28). Recently, there has been increased attention of the IGF signaling pathway from a therapeutic perspective, as monoclonal antibodies and small molecules targeting the IGF1R became available in the last few years (29). It was previously reported that the majority of mantle cell lymphoma cell lines and primary cases exhibited elevated IMP3 mRNA expression levels, and a that subset also expressed IGF1R (30). In order to determine the underlying mechanisms of IMP3 in GEP-NEN progression, the association between the expression of IMP3 and IGF1R was analyzed, as well as for the invasion-associated proteins MMP2 and CD44. The results demonstrated that IMP3 expression was positively correlated with IGFR1 and MMP2, indicating that IMP3 may activate the IGF1R signaling pathway and promote the proliferation of tumor cells by enhancing the expression of IGFII, and that it may also participate in the invasion and metastasis of GEP-NEN by MMP2 indirectly. IMP3 and CD44 were negatively correlated, but this was not statistically significant, which indicates that CD44 may not be a key molecule involved in GEP-NET invasion and metastasis.

In conclusion, in the present study, IMP3 was demonstrated to be frequently expressed in GEP-NEN. IMP3 staining may increase the accuracy of a histological diagnosis and of tumor grading, and may facilitate the identification of patients with a poor prognosis and who may be at higher risk of recurrence in high-grade GEP-NEN. IMP3 may be a potential immunohistochemical biomarker able to be used to evaluate the tumor progression and prognosis of GEP-NEN. However, identification of the underlying mechanisms of IMP3 in GEP-NEN requires further investigation.

\section{Acknowledgements}

The present study was supported by the National Natural Science Foundation of China (grant nos. 81072033 and 81372580), the Natural Science Foundation of Hebei Province (grant no. C2010000619), the Key Medical Research Program of Hebei (grant nos. ZL20140022 and 20160620) and the Health Department of Hebei Province (grant no. 20110460).

\section{References}

1. Yao JC, Hassan M, Phan A, Dagohoy C, Leary C, Mares JE, Abdalla EK, Fleming JB, Vauthey JN, Rashid A and Evans DB: One hundred years after 'carcinoid': Epidemiology of and prognostic factors for neuroendocrine tumors in 35,825 cases in the United States. J Clin Oncol 26: 3063-3072, 2008.

2. Niederle MB, Hackl M, Kaserer K and Niederle B: Gastroenteropancreatic neuroendocrine tumours: The current incidence and staging based on the WHO and European Neuroendocrine Tumour Society classification: An analysis based on prospectively collected parameters. Endocr Relat Cancer 17: 909-918, 2010.

3. Massironi S, Sciola V, Peracclli M, Ciafardini C, Spampatti MP and Conte D: Neuroendocrine tumors of the gastro-entero-pancreatic system. World J Gasnoenterol 14: 5377-5384, 2008.

4. Pape UF, Berndt U, Müller-Nordhorn J, Böhmig M, Roll S, Koch M, Willich SN and Wiedenmann B: Prognostic factors of long-term outcome in gastroenteropancreatic neuroendocrine tumours. Endocr Relat Cancer 15: 1083-1097, 2008.

5. Fitzgerald TL, Hickner ZJ, Schmitz M and Kort EJ: Changing incidence of pancreatic neoplasms: A 16-year review of statewide tumor registry. Pancreas 37: 134-138, 2008.

6. Nielsen FC, Nielsen J and Christiansen J: A family of IGF-II mRNA binding proteins (IMP) involved in RNA trafficking. Scand J Clin Lab Invest Suppl 234: 93-99, 2001.

7. Nielsen J, Christiansen J, Lykke-Andersen J, Johnsen AH, Wewer UM and Nielsen FC: A family of insulin like growth factor II mRNA-binding proteins represses translation in late development. Mol Cell Biol 19: 1262-1270, 1999.

8. Mueller-Pillasch F, Pohl B, Wilda M, Lacher U, Beil M, Wallrapp C, Hameister H, Knöchel W, Adler G and Gress TM: Expression of the highly conserved RNA binding protein KOC in embryogenesis. Mech Dev 88: 95-99, 1999. 
9. Yaniv K and Yisraeli JK: The involvement of a conserved family of RNA binding proteins in embryonic development and carcinogenesis. Gene 287: 49-54, 2002.

10. Okada K, Fujiwara Y, Nakamura Y, Takiguchi S, Nakajima K, Miyata H, Yamasaki M, Kurokawa Y, Takahashi T, Mori M and Doki Y: Oncofetal protein, IMP3, a potential marker for prediction of postoperative peritoneal dissemination in gastric adenocarcinoma. J Surg Oncol 105: 780-785, 2012.

11. Li D, Yan D, Tang H, Zhou C, Fan J, Li S, Wang X, Xia J, Huang F, Qiu G and Peng Z: IMP3 is a novel prognostic marker that correlates with colon cancer progression and pathogenesis. Ann Surg Oncol 16: 3499-3506, 2009.

12. Beljan Perak R, Durdov MG, Capkun V, Ivcevic V, Pavlovic A Soljic V and Peric M: IMP3 can predict aggressive behaviour of lung adenocarcinoma. Diagn Pathol 7: 165, 2012.

13. Liao B, Hu Y and Brewer G: RNA-binding protein insulin-like growth factor mRNA-binding protein 3 (IMP-3) promotes cell survival via insulin-like growth factor II signaling after ionizing radiation. J Biol Chem 286: 31145-31152, 2011.

14. Suvasini R, Shruti B, Thota B, Shinde SV, Friedmann-Morvinski D, Nawaz Z, Prasanna KV, Thennarasu K, Hegde AS, Arivazhagan A, et al: Insulin growth factor-2 binding protein 3 (IGF2BP3) is a glioblastoma-specific marker that activates phosphatidylinositol 3-kinase/mitogen-activated protein kinase (PI3K/MAPK) pathways by modulating IGF-2. J Biol Chem 286: 25882-25890, 2011.

15. You L, Liu C, Tang H, Liao Y and Fu S: Advances in targeting insulin-like growth factor signaling pathway in cancer treatment. Curr Pharm Des 20: 2899-2911, 2014

16. Singh P, Alex JM and Bast F: Insulin receptor (IR) and insulin-like growth factor receptor 1 (IGF-1R) signaling systems: Novel treatment strategies for cancer. Med Oncol 31: 805, 2014.

17. Bruchim I, Sarfstein R and Werner H: The IGF hormonal network in endometrial cancer: Functions, regulation, and targeting approaches. Front Endocrinol (Lausanne) 5: 76, 2014

18. Findeis-Hosey JJ and $\mathrm{Xu} \mathrm{H}$ : The use of insulin like-growth factor II messenger RNA binding protein-3 in diagnostic pathology. Hum Pathol 42: 303-314, 2011.

19. Bosman FT, Carneiro F, Hruban RH, et al: WHO classification of tumours of the digestive system. 4. Lyon: The International Agency for Research on Cancer 2010.

20. Klöppel G, Rindi G, Perren A, Komminoth P and Klimstra DS The ENETS and AJCC/UICC TNM classifications of the neuroendocrine tumors of the gastrointestinal tract and the pancreas: A statement. Virchows Arch 456: 595-597, 2010
21. Wang L, Li HG, Xia ZS, Lü J and Peng TS: IMP3 is a novel biomarker to predict metastasis and prognosis of gastric adenocarcinoma: A retrospective study. Chin Med J (Engl) 123: 3554-3558, 2010.

22. Li HG, Han JJ, Huang ZQ, Wang L, Chen WL and Shen XM: IMP3 is a novel biomarker to predict metastasis and prognosis of tongue squamous cell carcinoma. J Craniofac Surg 22: 2022-2025, 2011

23. Findeis-Hosey $\mathrm{JJ}$ and $\mathrm{Xu} \mathrm{H}$ : Insulin-like growth factor II-messenger RNA-binding protein-3 and lung cancer. Biotech Histochem 87: 24-29, 2012.

24. Lu D, Yang X, Jiang NY, Woda BA, Liu Q, Dresser K, Mercurio AM, Rock KL and Jiang Z: IMP3, a new biomarker to predict progression of cervical intraepithelial neoplasia into invasive cancer. Am J Surg Pathol 35: 1638-1645, 2011.

25. Bell JL, Wächter K, Mühleck B, Pazaitis N, Köhn M, Lederer M and Hüttelmaier S: Insulin-like growth factor 2 mRNA-binding proteins (IGF2BPs): Post-transcriptional drivers of cancer progression? Cell Mol Life Sci 70: 2657-2675, 2013.

26. Del Gobbo A, Vaira V, Guerini Rocco E, Palleschi A, Bulfamante G, Ricca D, Fiori S, Bosari S and Ferrero S: The oncofetal protein IMP3: A useful marker to predict poor clinical outcome in neuroendocrine tumors of the lung. J Thorac Oncol 9: 1656-1661, 2014.

27. Pasiliao CC, Chang CW, Sutherland BW, Valdez SM, Schaeffer D, Yapp DT and Ng SS: The involvement of insulin-like growth factor 2 binding protein 3 (IMP3) in pancreatic cancer cell migration, invasion, and adhesion. BMC Cancer 15: 266, 2015.

28. Lederer M, Bley N, Schleifer C and Hüttelmaier S: The role of the oncofetal IGF2 mRNA-binding protein 3 (IGF2BP3) in cancer. Semin Cancer Biol 29: 3-12, 2014.

29. Bruchim I and Werner H: Targeting IGF-1 signaling pathways in gynecologic malignancies. Expert Opin Ther Targets 17: 307-320, 2013.

30. Hartmann EM, Beà S, Navarro A, Trapp V, Campo E, Ott G and Rosenwald A: Increased tumor cell proliferation in mantle cell lymphoma is associated with elevated insulin-like growth factor 2 mRNA-binding protein 3 expression. Mod Pathol 25: 1227-1235, 2012 